\title{
Recent Advances in Mode of Action and Biosynthesis Studies of the Clinically Used Antibiotic Fidaxomicin
}

\author{
Andrea Dorst, Erik Jung, and Karl Gademann* \\ $\S S C S-D S M$ Award for best poster presentation in Medicinal Chemistry
}

\begin{abstract}
The natural product antibiotic fidaxomicin is a marketed drug for the treatment of bacterial infections in the gut. Due to its promising in vitro activities against Mycobacterium tuberculosis, the development of next generation fidaxomicin analogs is of great interest. This article reviews the most recent advances, including the elucidation of a unique mode of action by cryo-EM structures, and the efforts towards the clarification of the biosynthetic pathway. Moreover, known fidaxomicin analogs and their reported antibacterial activities are summarized.
\end{abstract}

Keywords: Antibiotics · Biosynthesis · Mode of action · Natural products · SAR studies

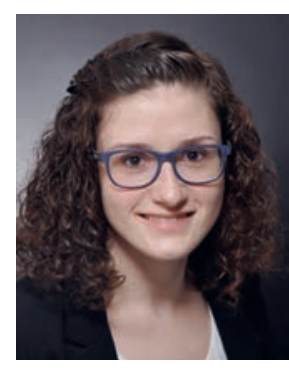

Andrea Dorst received her Bachelor and Master's degrees in chemistry from the University of Basel. She performed her Master thesis in the research group of Prof. Dr. Karl Gademann on total synthesis. After receiving her Master's degree, she stayed in the Gademann group, that just had moved to the University of Zurich, to pursue her PhD studies on the synthesis of semisynthetic antibiotics.

\section{Introduction}

Fidaxomicin (1) is the first member of a class of antibiotics possessing an 18-membered, highly unsaturated macrolactone.[1] Fidaxomicin was first isolated in 1972 as lipiarmycin A3 by Gruppo Lepetit scientists from an actinobacterium along with similar natural products. ${ }^{[2]}$ Interestingly, further bacterial strains were later found to produce similar compounds which were named clostomicins and tiacumicins, respectively. ${ }^{[3]}$ Extensive structural studies revealed that lipiarmycin A3, clostomicin B1, and tiacumicin B are identical. ${ }^{[3 \mathrm{a}, 4]}$ Moreover, these studies identified the rhamnoside-dichlorohomoorsellinate moiety as well as the modified noviose as important structural features (Fig. 1). ${ }^{[5]}$ Notably, the stereogenic center at C(18) was under discussion for a long time and it was assumed that lipiarmycin $\mathrm{A} 3$ and tiacumicin $B$ distinguished themselves by the configuration of the $C(18)$ stereocenter. ${ }^{[6]}$ However, total synthesis campaigns, ${ }^{[7]}$ extensive NMR analysis, and finally an X-ray analysis of these two natural products unambiguously verified their co-identity and revealed the $(R)$-configuration. ${ }^{[4]}$

Fidaxomicin (1) was reported to be active mainly against Gram-positive bacteria including strains not susceptible to other antibiotics. Excellent activities were reported against Clostridium difficile (recently reclassified as Clostridioides difficile $^{[8]}$ and Clostridium perfringens as well as resistant strains of Staphylococcus aureus and Mycobacterium tuberculosis. ${ }^{[3, \mathrm{a}, 9]}$ No cross-resistance was observed with a broad panel of anti-

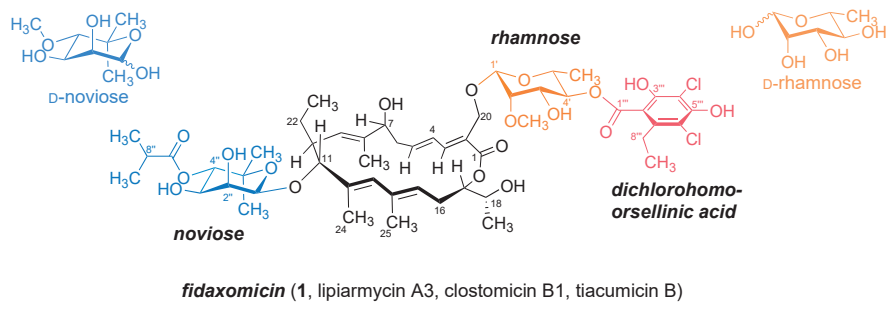

Fig. 1. Structure of the natural product fidaxomicin (1).

biotics. ${ }^{[2 b, 10]}$ Although possessing activity against a variety of different bacterial strains, the clinical use of fidaxomicin (1) is currently limited to the treatment of $C$. difficile infections (CDI). The FDA approved the antibiotic $\mathbf{1}$ in 2011 for the treatment of hospital-acquired diarrhea caused by $C$. difficile and it was introduced to the market as Dificlir ${ }^{\circledR}$. Due to its low water solubility and thus low systemic absorption, treatment of infections outside the gut is not yet feasible.[11]

Tuberculosis (TB) remains one of the top ten causes of death worldwide. The WHO estimated that around 10 million people were infected in 2018 and around 1.5 million people died as a consequence. ${ }^{[12]}$ Unfortunately, resistance is developing fast and spreads globally. In this context, fidaxomicin's potential to become a useful antibiotic for the treatment of severe infections outside the gut has not yet been exploited. Despite its unfavorable bioavailability, this natural product could be a promising starting point in the development of new antibiotics. In 2012, Zhu and coworkers excellently reviewed the early history of fidaxomicin from its discovery to the introduction to the market in 2011,[1a] and a recent review article highlighted the synthetic aspects. ${ }^{[7 \mathrm{~g}]}$ This article focuses on the more recent advances in the field with emphasis on new insights into the biosynthesis and mechanistic aspects from cryo-EM structures and includes a summary of the antibacterial activities of the known derivatives. A recent article summarizes the synthetic chemistry and biology of fidaxomicin. ${ }^{[1 d]}$ 


\section{Mode of Action}

The first report on the mode of action of fidaxomicin (1) appeared soon after its discovery. Therein, Parenti and coworkers reported that antibiotic $\mathbf{1}$ interferes with RNA synthesis by inhibiting bacterial RNA polymerase (RNAP). ${ }^{[13]}$ In the following decades, further progress in elucidation of the molecular basis of RNAP inhibition was achieved. ${ }^{[14]}$ Recently, cryo-electron microscopy (cryo-EM) structures of fidaxomicin binding to $M$. $t u$ berculosis RNAP, independently disclosed by the research groups of Campbell and Ebright, respectively, revealed the actual mechanism of action. ${ }^{[15]}$ The bacterial RNAP core enzyme consists of five subunits $\left(\alpha \alpha \beta \beta^{\prime} \omega\right)$ and an initiation factor $\sigma$ (Scheme 1).

Upon binding of the $\sigma$-factor, the so-called holoenzyme is formed.[16] During initiation of the transcription, the $\sigma$-factor recognizes the -35 and -10 promoter sequences of the DNA by undergoing a conformational change from an open to a closed clamp state. Next, the double-stranded DNA is melted to form an open promoter complex $\left(\mathrm{RP}_{\mathrm{O}}\right)$ onto which nucleotides can adhere to build up a new RNA strand. The cryo-EM structure revealed that fidaxomicin undergoes interactions with the $\beta, \beta$ ' as well as $\sigma$-subunits at the switch-region. The switch-region is located at the base of the RNA clamp and mediates conformational changes. By binding to this region, fidaxomicin traps an open clamp state, thereby preventing simultaneous recognition of the -10 and -35 promoter elements by the $\sigma$-subunit and formation of the transcription bubble.

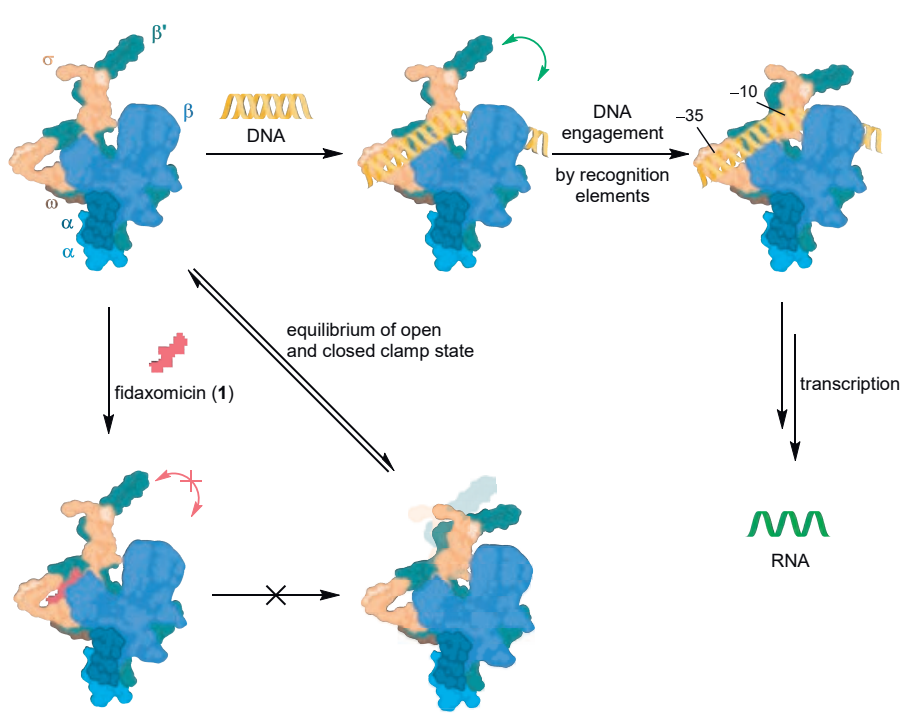

Scheme 1. Mode of action of RNA polymerase inhibition by fidaxomicin (1).

\section{Biosynthesis}

In 2011, Zhang and coworkers reported a first proposal for the biosynthetic pathway of fidaxomicin based on a genetic approach.[17] Using gene-knockout mutants, Zhang and coworkers isolated a great number of truncated fidaxomicin analogs which provided not only important information on involved enzymes and their functions, but also enabled a broad structure-activity relationship (SAR) study. They identified the biosynthetic gene cluster of $D$. aurantiacum subsp. hamdenensis, one of the known producer strains. Sequence analysis and extensive analysis of truncated products obtained from the fermentation of geneknockout mutants led to the proposal of a reasonable biosynthesis, which was refined in following years. ${ }^{[18]}$ Four genes (tiaA1-A4) were found to encode for a modular type I polyketide synthase (PKS) that generates the aglycon 2 starting from malonyl-CoA and derivatives thereof (Scheme 2). Next, the $\mathrm{C}(20)$-position is oxidized by a cytochrome P450 (TiaP2) followed by noviosylation of $\mathrm{OH}-\mathrm{C}(11)$ catalyzed by glycosyltransferase TiaG1 to give monoglycosylated $\mathbf{4}$. Subsequently, the rhamnosylation is mediated by glycosyltransferase TiaG2 and acyltransferase TiaS6 installs the isobutyric ester moiety on the OH-C(4") on the noviose. However, the catalytic order of TiaS6 and TiaG2 is not yet determined. Next, the homoorsellinic acid moiety $\mathbf{8}$, which is biosynthesized by a type I PKS from a propionyl-CoA starter unit and three malonyl-CoA extender units, is coupled to rhamnose $\mathrm{OH}-\mathrm{C}\left(4^{\prime}\right)$ by acyltransferase TiaF. The halogenase TiaM installs the chlorine substituents to the aryl 6 and methylation at $\mathrm{OH}-\mathrm{C}\left(2^{\prime}\right)$ occurs, however, the reaction timing remains elusive. As the last step in the biosynthesis, the oxidation in C(18)-position by TiaP1 was ascertained via extensive kinetic studies. ${ }^{[18 c]}$

\section{Structure-Activity Relationship Studies}

Structure-activity relationship (SAR) studies are of great importance when considering the generation of new fidaxomicin analogs with improved pharmacokinetic properties. Fig. 2 summarizes all isolated, naturally occurring fidaxomicin analogs $[2,3,5,19]$ as well as derivatives obtained by fermentation of gene-knockout mutants which produced truncated fidaxomicin analogs missing the noviose and/or the rhamnose moieties. ${ }^{[17,18]}$ Semisynthetically synthesized analogs are listed, but their biological activity is omitted due to lack of comparable biological data. ${ }^{[15 b, 20]}$ Antibacterial activity has not been reported for all compounds and the existing data is difficult to compare due to the utilization of different assays and/or bacterial strains. Nevertheless, a general trend is observable. Accordingly, the noviose and rhamnose sugar moiety are crucial for the antibacterial activity of $\mathbf{1}$. Analogs missing one or both of these moieties completely lose their antibacterial

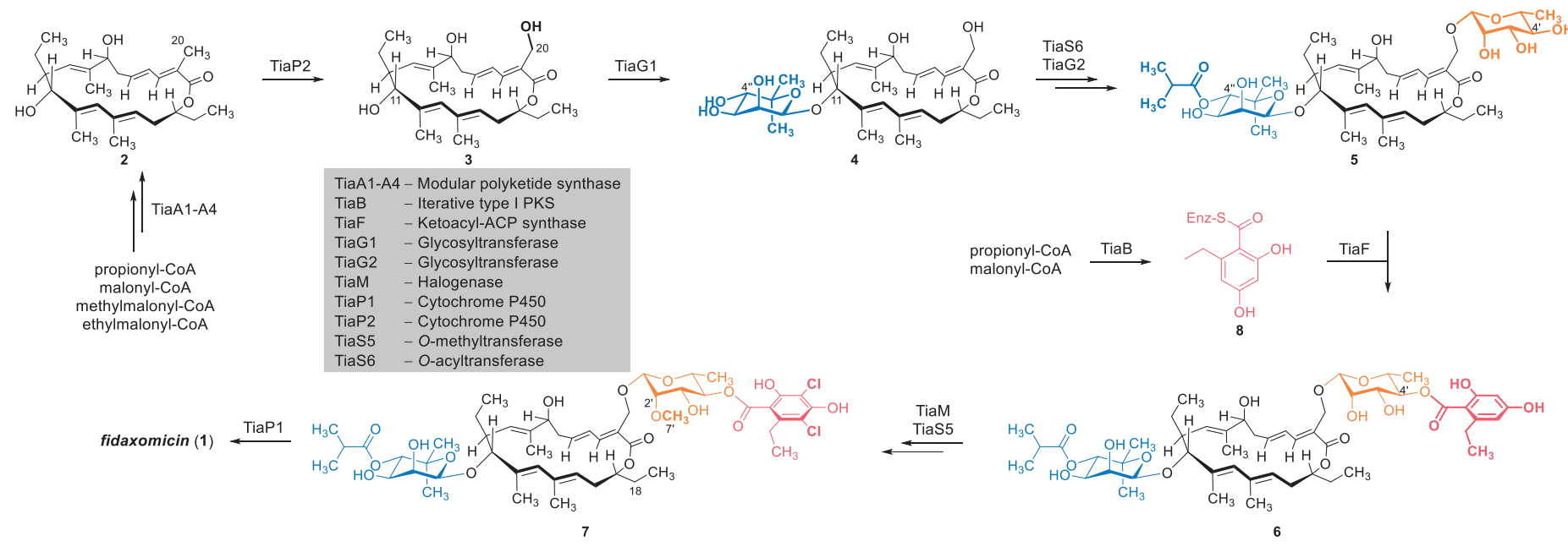

Scheme 2. Proposed biosynthetic pathway of fidaxomicin (1). 


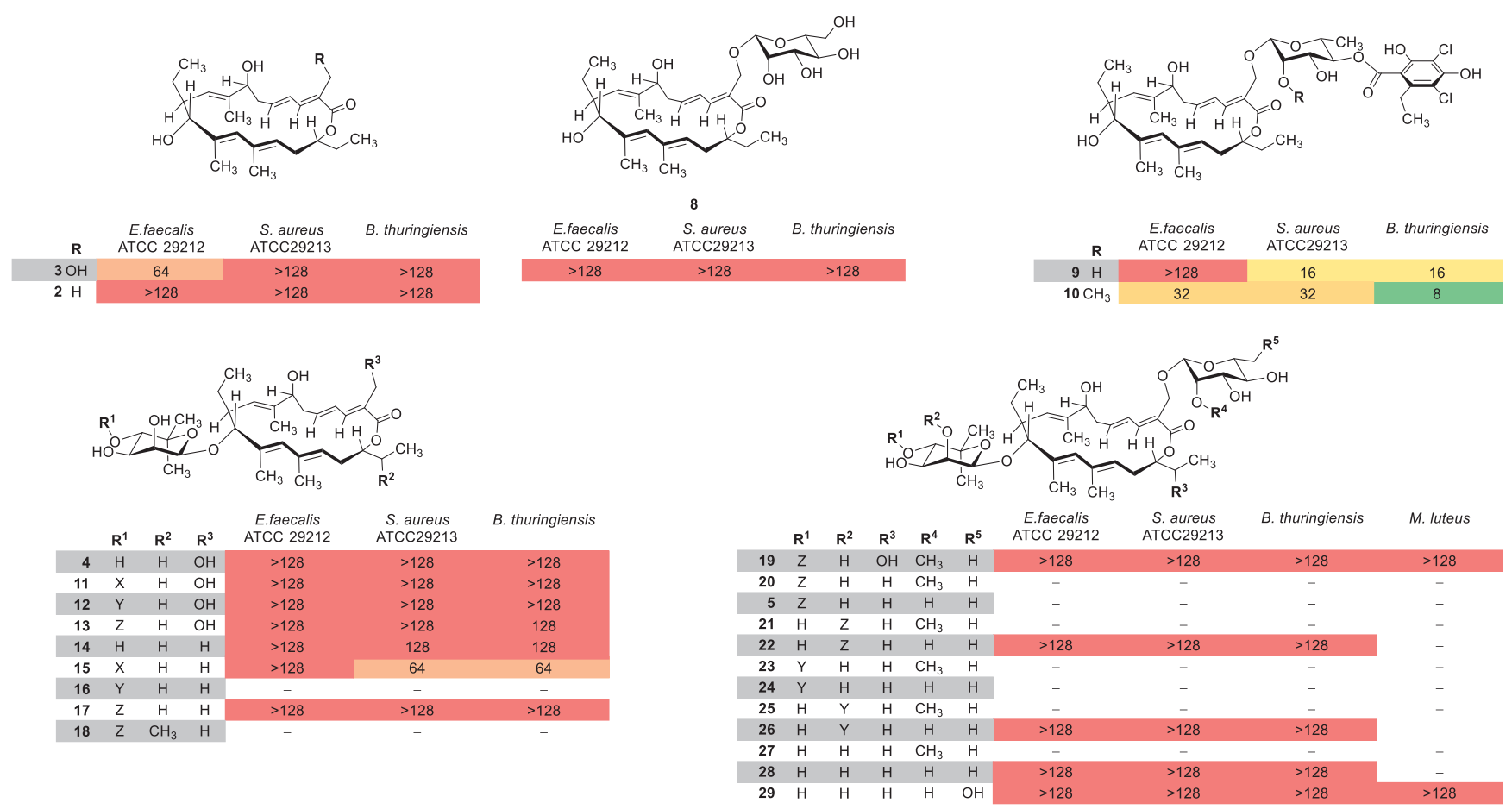

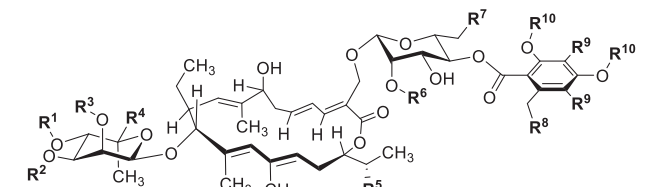

$\begin{array}{llllllllllllll}\mathbf{R}^{1} & \mathbf{R}^{2} & \mathbf{R}^{3} & \mathbf{R}^{4} & \mathbf{R}^{5} & \mathbf{R}^{6} & \mathbf{R}^{7} & \mathbf{R}^{8} & \mathbf{R}^{9} & \mathbf{R}^{10} & \begin{array}{c}\text { Ef faecalis } \\ \text { ATCC } 29212\end{array} & \begin{array}{c}\text { S. aureus } \\ \text { ATCC29213 }\end{array} & \text { B. thuringiensis } & \text { M. luteus }\end{array}$

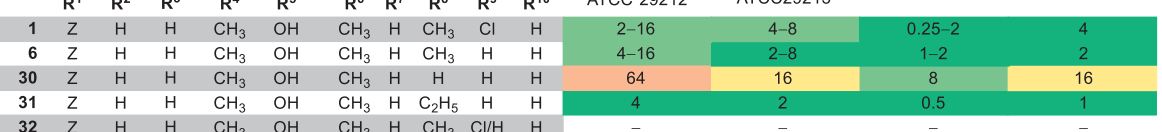

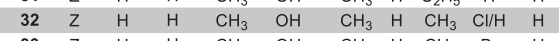

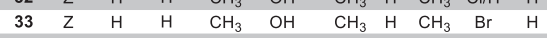

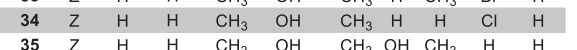

$\begin{array}{lllllllllll}35 & \mathrm{Z} & \mathrm{H} & \mathrm{H} & \mathrm{CH}_{3} & \mathrm{OH} & \mathrm{CH}_{3} & \mathrm{OH} & \mathrm{CH}_{3} & \mathrm{H} & \mathrm{H} \\ 36 & \mathrm{Z} & \mathrm{H} & \mathrm{H} & \mathrm{CH}_{3} & \mathrm{OH} & \mathrm{H} & \mathrm{H} & \mathrm{CH}_{3} & \mathrm{Cl} & \mathrm{H}\end{array}$

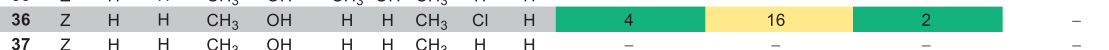

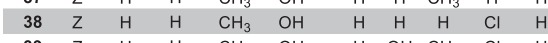

$\begin{array}{lllllllllll}39 & \mathrm{Z} & \mathrm{H} & \mathrm{H} & \mathrm{CH}_{3} & \mathrm{OH} & \mathrm{H} & \mathrm{OH} & \mathrm{CH}_{3} & \mathrm{Cl} & \mathrm{H}\end{array}$

$\begin{array}{ccccccccccc}7 & \mathrm{Z} & \mathrm{H} & \mathrm{H} & \mathrm{CH}_{3} & \mathrm{H} & \mathrm{CH}_{3} & \mathrm{H} & \mathrm{CH}_{3} & \mathrm{Cl} & \mathrm{H} \\ 40 & \mathrm{Z} & \mathrm{H} & \mathrm{H} & \mathrm{CH}_{3} & \mathrm{H} & \mathrm{CH}_{3} & \mathrm{H} & \mathrm{CH}_{3} & \mathrm{H} & \mathrm{H}\end{array}$

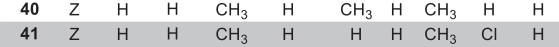

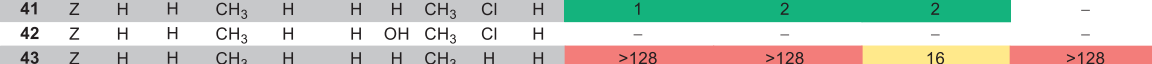

$\begin{array}{llllllllllll}43 & \mathrm{Z} & \mathrm{H} & \mathrm{H} & \mathrm{CH}_{3} & \mathrm{H} & \mathrm{H} & \mathrm{H} & \mathrm{CH}_{3} & \mathrm{H} & \mathrm{H} \\ 44 & \mathrm{Z} & \mathrm{H} & \mathrm{H} & \mathrm{CH}_{3} & =\mathrm{O} & \mathrm{CH}_{3} & \mathrm{H} & \mathrm{CH}_{3} & \mathrm{Cl} & \mathrm{H}\end{array}$

$\begin{array}{lllllllllll}44 & \mathrm{Z} & \mathrm{H} & \mathrm{H} & \mathrm{CH}_{3} & =0 & \mathrm{CH}_{3} & \mathrm{H} & \mathrm{CH}_{3} & \mathrm{Cl} & \mathrm{H} \\ 45 & \mathrm{Z} & \mathrm{H} & \mathrm{H} & \mathrm{CH}_{3} & =\mathrm{O} & \mathrm{CH}_{3} & \mathrm{H} & \mathrm{CH}_{3} & \mathrm{H} & \mathrm{H}\end{array}$

$\begin{array}{lllllllllllll}46 & \mathrm{Z} & \mathrm{H} & \mathrm{H} & \mathrm{CH}_{3} & \mathrm{CH}_{3} & \mathrm{H} & \mathrm{H} & \mathrm{CH}_{3} & \mathrm{Cl} & \mathrm{H}\end{array}$

$\begin{array}{lllllllllll}47 & \mathrm{Z} & \mathrm{H} & \mathrm{H} & \mathrm{CH}_{3} & \mathrm{CH}_{3} & \mathrm{CH}_{3} & \mathrm{H} & \mathrm{CH}_{3} & \mathrm{Cl} & \mathrm{H}\end{array}$

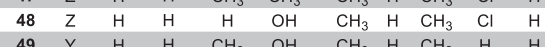

$\begin{array}{llllllllllll}49 & \mathrm{Y} & \mathrm{H} & \mathrm{H} & \mathrm{CH}_{3} & \mathrm{OH} & \mathrm{CH}_{3} & \mathrm{H} & \mathrm{CH}_{3} & \mathrm{H} & \mathrm{H} \\ \mathbf{5 0} & \mathrm{Y} & \mathrm{H} & \mathrm{H} & \mathrm{CH}_{3} & \mathrm{OH} & \mathrm{H} & \mathrm{H} & \mathrm{CH}_{3} & \mathrm{Cl} & \mathrm{H}\end{array}$

$\begin{array}{lllllllllll}51 & \mathrm{Y} & \mathrm{H} & \mathrm{H} & \mathrm{CH}_{3} & \mathrm{H} & \mathrm{CH}_{3} & \mathrm{H} & \mathrm{CH}_{3} & \mathrm{Cl} & \mathrm{H} \\ 52 & \mathrm{Y} & \mathrm{H} & \mathrm{H} & \mathrm{CH}_{3} & \mathrm{H} & \mathrm{H} & \mathrm{H} & \mathrm{CH}_{3} & \mathrm{Cl} & \mathrm{H}\end{array}$

$\begin{array}{lllllllllll}52 & \mathrm{Y} & \mathrm{H} & \mathrm{H} & \mathrm{CH}_{3} & \mathrm{H} & \mathrm{H} & \mathrm{H} & \mathrm{CH}_{3} & \mathrm{Cl} & \mathrm{H} \\ 53 & \mathrm{X} & \mathrm{H} & \mathrm{H} & \mathrm{CH}_{3} & \mathrm{OH} & \mathrm{CH}_{3} & \mathrm{H} & \mathrm{CH}_{3} & \mathrm{H} & \mathrm{H}\end{array}$

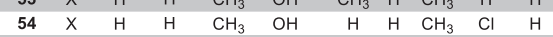

$\begin{array}{llllllllllllllll}55 & \mathrm{X} & \mathrm{H} & \mathrm{H} & \mathrm{CH}_{3} & \mathrm{H} & \mathrm{CH}_{3} & \mathrm{H} & \mathrm{CH}_{3} & \mathrm{Cl} & \mathrm{H}\end{array}$

$\begin{array}{lllllllllll}56 & \mathrm{X} & \mathrm{H} & \mathrm{H} & \mathrm{CH}_{3} & \mathrm{H} & \mathrm{H} & \mathrm{H} & \mathrm{CH}_{3} & \mathrm{Cl} & \mathrm{H} \\ 57 & \mathrm{H} & \mathrm{H} & \mathrm{H} & \mathrm{CH}_{3} & \mathrm{OH} & \mathrm{CH}_{3} & \mathrm{H} & \mathrm{CH}_{3} & \mathrm{Cl} & \mathrm{H}\end{array}$

$\begin{array}{lllllllllll}57 & \mathrm{H} & \mathrm{H} & \mathrm{H} & \mathrm{CH}_{3} & \mathrm{OH} & \mathrm{CH}_{3} & \mathrm{H} & \mathrm{CH}_{3} & \mathrm{Cl} & \mathrm{H} \\ 58 & \mathrm{H} & \mathrm{H} & \mathrm{H} & \mathrm{CH}_{3} & \mathrm{OH} & \mathrm{CH}_{3} & \mathrm{H} & \mathrm{CH}_{3} & \mathrm{H} & \mathrm{H}\end{array}$

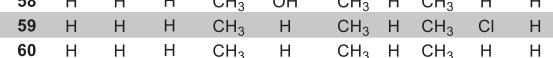

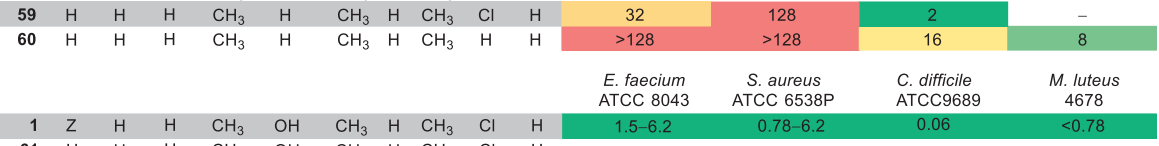

$\begin{array}{rrrrrrrrrrr}1 & \mathrm{Z} & \mathrm{H} & \mathrm{H} & \mathrm{CH}_{3} & \mathrm{OH} & \mathrm{CH}_{3} & \mathrm{H} & \mathrm{CH}_{3} & \mathrm{Cl} & \mathrm{H} \\ 61 & \mathrm{H} & \mathrm{H} & \mathrm{H} & \mathrm{CH}_{3} & \mathrm{OH} & \mathrm{CH}_{3} & \mathrm{H} & \mathrm{CH}_{3} & \mathrm{Cl} & \mathrm{H}\end{array}$

$\begin{array}{cccc}\text { ATCC 8043 } & \text { ATCC 6538P } & \text { ATCC9689 } & 4678 \\ 1.5-6.2 & 0.78-6.2 & 0.06 & <0.78\end{array}$

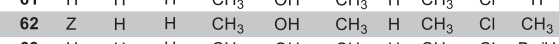

$\begin{array}{llllllllllll}63 & \mathrm{H} & \mathrm{H} & \mathrm{H} & \mathrm{CH}_{3} & \mathrm{OH} & \mathrm{CH}_{3} & \mathrm{H} & \mathrm{CH}_{3} & \mathrm{Cl} & \mathrm{Bn} / \mathrm{H}\end{array}$

$\begin{array}{lllllllllll}64 & \mathrm{H} & \mathrm{H} & \mathrm{Z} & \mathrm{CH}_{3} & \mathrm{OH} & \mathrm{CH}_{3} & \mathrm{H} & \mathrm{CH}_{3} & \mathrm{Cl} & \mathrm{H}\end{array}$

$\begin{array}{lllllllllll}65 & \mathrm{H} & \mathrm{Z} & \mathrm{H} & \mathrm{CH}_{3} & \mathrm{OH} & \mathrm{CH}_{3} & \mathrm{H} & \mathrm{CH}_{3} & \mathrm{Cl} & \mathrm{H} \\ 66 & \mathrm{H} & \mathrm{H} & \mathrm{Y} & \mathrm{CH}_{3} & \mathrm{OH} & \mathrm{CH}_{3} & \mathrm{H} & \mathrm{CH}_{3} & \mathrm{Cl} & \mathrm{H}\end{array}$

$\begin{array}{lllllllllll}66 & \mathrm{H} & \mathrm{H} & \mathrm{Y} & \mathrm{CH}_{3} & \mathrm{OH} & \mathrm{CH}_{3} & \mathrm{H} & \mathrm{CH}_{3} & \mathrm{Cl} & \mathrm{H} \\ 67 & \mathrm{X} & \mathrm{H} & \mathrm{H} & \mathrm{CH}_{3} & \mathrm{OH} & \mathrm{CH}_{3} & \mathrm{H} & \mathrm{CH}_{3} & \mathrm{Cl} & \mathrm{H}\end{array}$

$\begin{array}{lllllllllll}67 & \mathrm{X} & \mathrm{H} & \mathrm{H} & \mathrm{CH}_{3} & \mathrm{OH} & \mathrm{CH}_{3} & \mathrm{H} & \mathrm{CH}_{3} & \mathrm{Cl} & \mathrm{H} \\ 68 & \mathrm{Y} & \mathrm{H} & \mathrm{H} & \mathrm{CH}_{3} & \mathrm{OH} & \mathrm{CH}_{3} & \mathrm{H} & \mathrm{CH}_{3} & \mathrm{Cl} & \mathrm{H}\end{array}$

$\begin{array}{lllllllllll}69 & \mathrm{~W} & \mathrm{H} & \mathrm{H} & \mathrm{CH}_{3} & \mathrm{OH} & \mathrm{CH}_{3} & \mathrm{H} & \mathrm{CH}_{3} & \mathrm{Cl} & \mathrm{H}\end{array}$

$\begin{array}{lllllllllll}70 & \mathrm{H} & \mathrm{H} & \mathrm{Z} & \mathrm{CH}_{3} & \mathrm{OH} & \mathrm{CH}_{3} & \mathrm{H} & \mathrm{H} & \mathrm{Cl} & \mathrm{H}\end{array}$

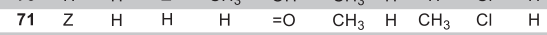

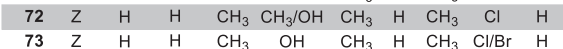

$\begin{array}{lllllllllll}73 & \mathrm{Z} & \mathrm{H} & \mathrm{H} & \mathrm{CH}_{3} & \mathrm{OH} & \mathrm{CH}_{3} & \mathrm{H} & \mathrm{CH}_{3} & \mathrm{Cl} / \mathrm{Br} & \mathrm{H} \\ 74 & \mathrm{Z} & \mathrm{H} & \mathrm{H} & \mathrm{CH}_{3} & =\mathrm{O} & \mathrm{CH}_{3} & \mathrm{H} & \mathrm{CH}_{3} & \mathrm{Cl} / \mathrm{Br} & \mathrm{H}\end{array}$

$\begin{array}{lllllllllll}75 & \mathrm{Z} & \mathrm{H} & \mathrm{H} & \mathrm{CH}_{3} & \mathrm{OH} & \mathrm{CH}_{3} & \mathrm{H} & \mathrm{CH}_{3} & \mathrm{Br} / \mathrm{H} & \mathrm{H}\end{array}$

$\begin{array}{cc}100 & 50 \\ 6.2 & 12.5 \\ 12.5 & 25\end{array}$

5
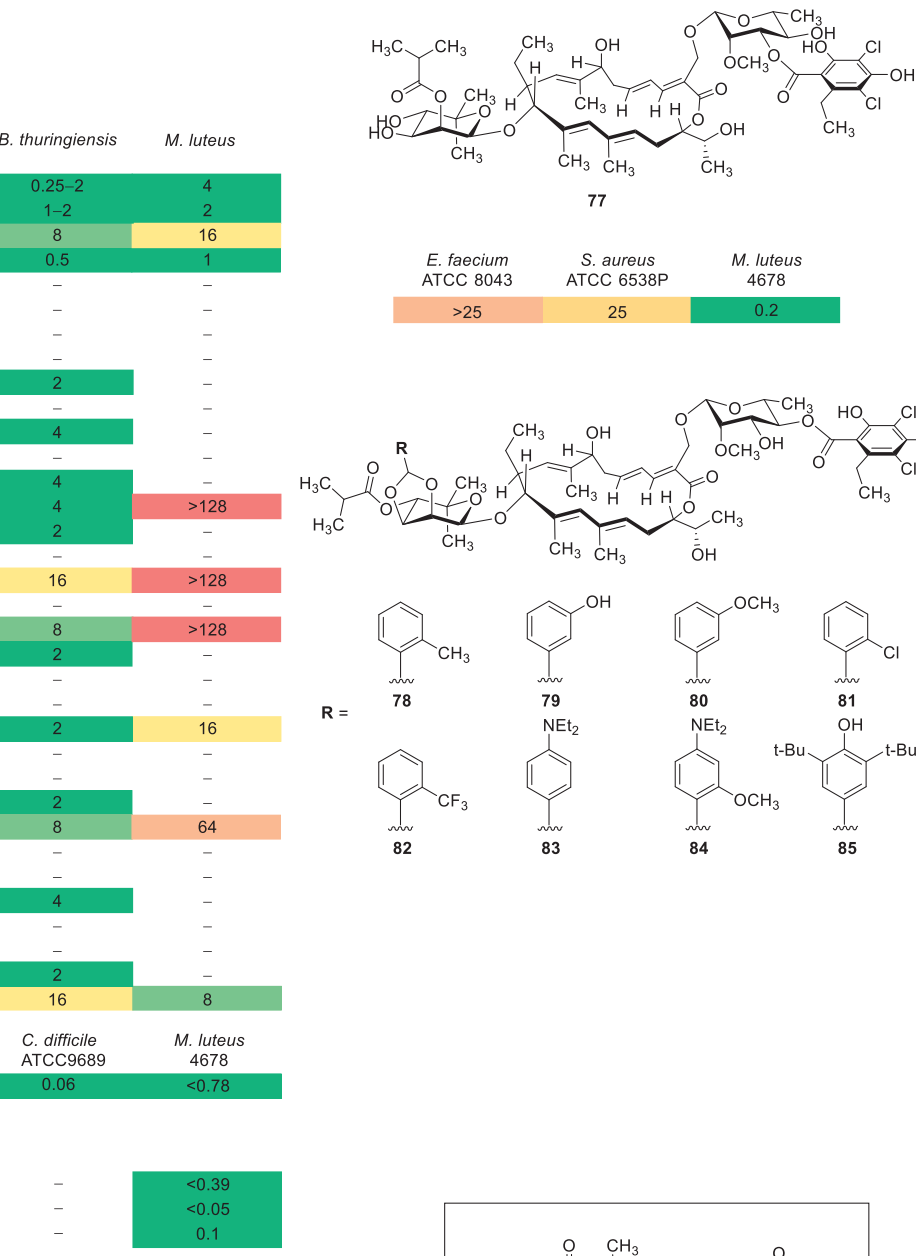

77

$\begin{array}{ccc}\text { E. faecium } & \text { S. aureus } & \text { M. luteus } \\ \text { ATCC } 8043 & \text { ATCC } 6538 \mathrm{P} & 4678\end{array}$

$\begin{array}{cccc}>25 & 25 & 0.2\end{array}$
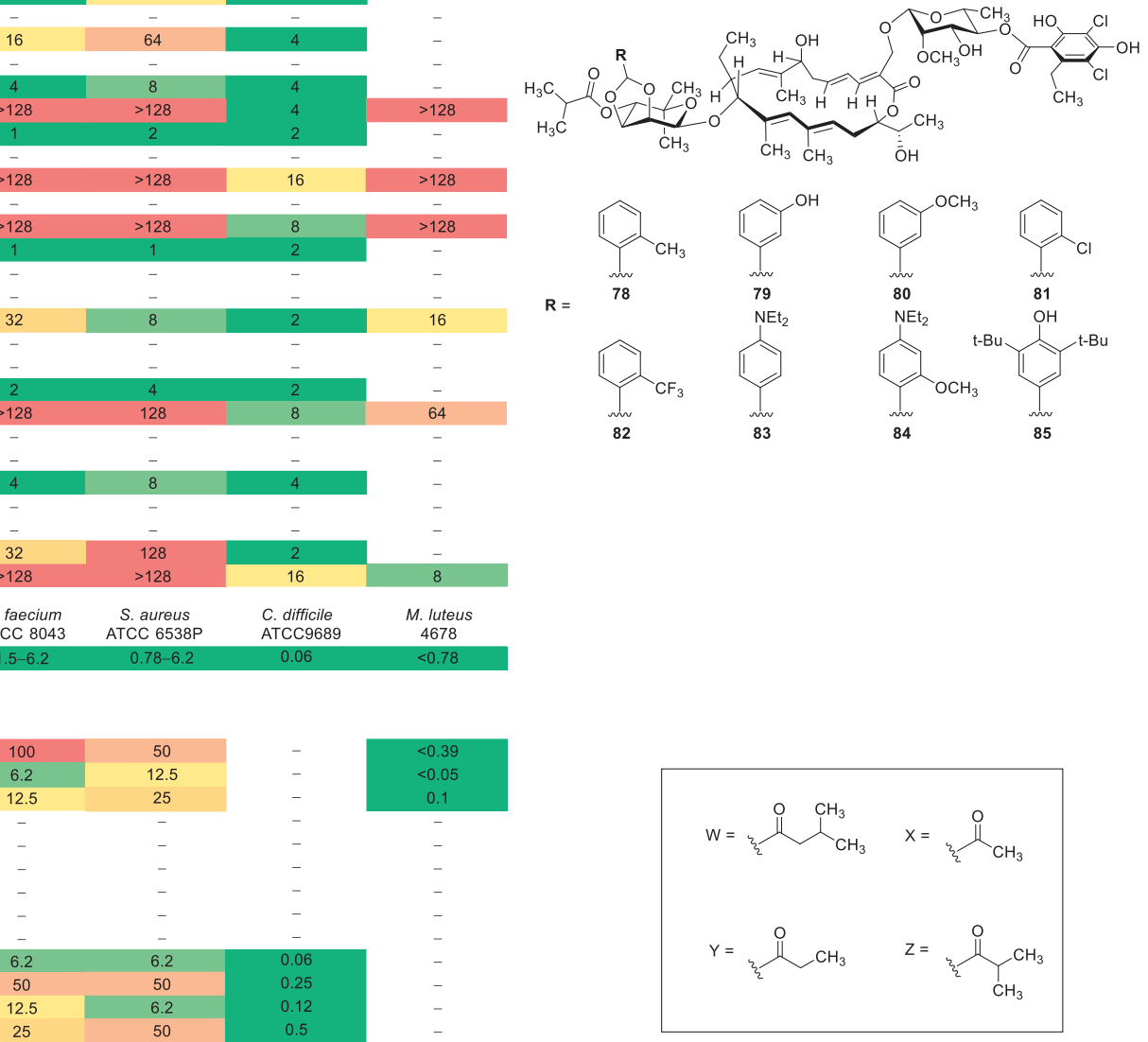

Fig. 2. Known fidaxomicin derivatives and their antibacterial activities given as minimum inhibitory concentrations (MIC, $\mu \mathrm{g} / \mathrm{mL})$. 
properties except for denoviosylated $\mathbf{9}$ and 10, which maintain some antibacterial activity even though significantly decreased compared to the parent compound 1. Noteworthy, a propyl (ana$\log 31$ ) instead of an ethyl (analog 6) or methyl group (analog 30) on the homoorsellinic acid with lacking chloride substituents is beneficial for the antibacterial activity.

Moreover, the isobutyric ester moiety on the noviose is crucial for its excellent activity. Hydrolysis of this isobutyric ester (61, OP-1118, known metabolite of fidaxomicin) leads to a more than 8 -fold decrease in activity against $C$. difficile. ${ }^{[21]}$ Replacement of isobutyric ester by propionic ester (49) or acetate (53) also diminishes the activity. Migration of the isobutyric ester to the $\mathrm{OH}-\mathrm{C}(2 ")(64)$ is not tolerated and results in 8-16-fold increase of the minimum inhibitory concentration (MIC) value, whereas $\mathrm{OH}-\mathrm{C}(3$ ") (65) largely retains activity. For the compounds lacking the $\mathrm{OH}-\mathrm{C}(18)$ group, the results are ambiguous. While fidaxomicin without the $\mathrm{OH}-\mathrm{C}(18)$ group (7) slightly loses activity, an analog lacking $\mathrm{OH}-\mathrm{C}(18)$ as well as $\mathrm{C}\left(7^{\prime}\right)$-methyl group (41) shows improved activities. However, the compound only lacking C(7')-methyl group (36) also shows decreased activity. In contrast, the influence of the chloride substituents on the homoorsellinic acid moiety $(\mathbf{6}, \mathbf{7 3}, \mathbf{7 5})$ only appears to have a small influence on the antibiotic activity of fidaxomicin (1).

\section{Conclusion and Outlook}

With regard to the current precarious situation due the emergence of antibiotic-resistant bacteria, new antibiotics are urgently needed. The synthesis of new fidaxomicin analogs with improved bioavailability might allow for the treatment of infections like tuberculosis. These recent insights into the biosynthesis as well as mechanistic processes enable the generation of new fidaxomicin antibiotics based on structural considerations.

\section{Acknowledgements}

We thank the SNSF (Grant No. 182043) for partial funding of this work, the Swiss Chemical Society and DSM for the poster award to A. D., and the Alfred Werner fund and Hoffmann-La Roche for master scholarships to E. J..

Received: February 14, 2020

[1] a) K. M. Mullane, S. Gorbach, Expert Rev. Anti. Infect. Ther. 2011, 9, 767, doi: 10.1586/eri.11.53; b) W. Erb, J. Zhu, Nat. Prod. Rep. 2013, 30, 161, doi: 10.1039/C2NP20080E; c) J. B. McAlpine, J. Antibiot. 2017, 70, 492, doi: 10.1038/ja.2016.157; d) A. Dorst, K. Gademann, Helv. Chim. Acta 2020, doi: 10.1002/hlca.202000038.

[2] a) F. Parenti, H. Pagani, G. Beretta, J. Antibiot. 1975, 28, 247, doi: 10.7164/ antibiotics.28.247; b) C. Coronelli, R. J. White, G. C. Lancini, F. Parenti, J. Antibiot. 1975, 28, 253, doi: 10.7164/antibiotics.28.253; c) C. Coronelli, F. Parenti, R. White, H. Pagani, US Patent Appl. No. US 3,978,211, 1976.

[3] a) S. Õmura, N. Imamura, R. Õiwa, H. Kuga, R. Iwata, R. Masuma, Y. Iwai, J. Antibiot. 1986, 39, 1407, doi: 10.7164/antibiotics.39.1407; b) Y. Takashi, Y. Iwai, S. Õmura, J. Antibiot. 1986, 39, 1413, doi: 10.7164/ antibiotics.39.1413; c) R. J. Theriault, J. P. Karwowski, M. Jackson, R. L. Girolami, G. N. Sunga, C. M. Vojtko, L. J. Coen, J. Antibiot. 1987, 40, 567, doi: 10.7164/antibiotics.40.567; d) J. E. Hochlowski, S. J. Swanson, L. M. Ranfranz, D. N. Whittern, A. M. Buko, J. B. McAlpine, J. Antibiot. 1987, 40, 575, doi: 10.7164/antibiotics.40.575; e) Y. Ichikawa, Y.-H. Chiu, Y.-K. Shue, F. K. Babakhani, US Patent Appl. No. US 8,044,030 B2, 2008; f) M. Kurabachew, S. H. J. Lu, P. Krastel, E. K. Schmitt, B. L. Suresh, A. Goh, J. E. Knox, N. L. Ma, J. Jiricek, D. Beer, M. Cynamon, F. Petersen, V. Dartois, T. Keller, T. Dick, V. K. Sambandamurthy, J. Antimicrob. Chemother. 2008, 62, 713, doi: 10.1093/jac/dkn269.

[4] a) S. Serra, L. Malpezzi, A. Bedeschi, C. Fuganti, P. Fonte, Antibiotics 2017, 6, 7, doi: 10.3390/antibiotics6010007; b) A. Bedeschi, P. Fonte, G. Fronza, C. Fuganti, S. Serra, Nat. Prod. Commun. 2015, 11, 569, doi: 10.1177/1934578X1601100501.

[5] E. Martinelli, L. Faniuolo, G. Tuan, G. G. Gallo, B. Cavalleri, J. Antibiot 1983, 36, 1312, doi: 10.7164/antibiotics.36.1312.

[6] Y.-K. Shue, C.-K. Hwang, Y.-H. Chiu, A. Romero, F. Babakhani, F. Okumu, WO Patent Appl. No. WO 2006/085838 A1, 2005.
[7] a) F. Glaus, K. H. Altmann, Angew. Chem. Int. Ed. 2015, 54, 1937, doi: 10.1002/anie.201409510; b) W. Erb, J. M. Grassot, D. Linder, L. Neuville, J. Zhu, Angew. Chem. Int. Ed. 2015, 54, 1929, doi: 10.1002/anie.201409475; c) H. Miyatake-Ondozabal, E. Kaufmann, K. Gademann, Angew. Chem. Int. Ed. 2015, 54, 1933, doi: 10.1002/anie.201409464; d) E. Kaufmann, H. Hattori, H. Miyatake-Ondozabal, K. Gademann, Org. Lett. 2015, 17, 3514, doi: 10.1021/acs.orglett.5b01602; e) L. Jeanne-Julien, G. Masson, E. Astier, G. Genta-Jouve, V. Servajean, J.-M. Beau, S. Norsikian, E. Roulland, J. Org. Chem. 2018, 83, 921, doi: 10.1021/acs.joc.7b02909; f) L. Jeanne-Julien, G. Masson, E. Astier, G. Genta-Jouve, V. Servajean, J. M. Beau, S. Norsikian, E. Roulland, Org. Lett. 2017, 19, 4006, doi: 10.1021/acs.orglett.7b01744; g) E. Roulland, Synthesis 2018, 50, 4189, doi: 10.1055/s-0037-1609933; h) S. Norsikian, C. Tresse, M. François-Eude, L. Jeanne-Julien, G. Masson, V. Servajean, G. Genta-Jouve, J.-M. Beau, E. Roulland, Angew. Chem. Int. Ed. 2020, doi: 10.1002/anie.202000231.

[8] P. A. Lawson, D. M. Citron, K. L. Tyrrell, S. M. Finegold, Anaerobe 2016, 40, 95, doi: 10.1016/j.anaerobe.2016.06.008.

[9] a) R. N. Swanson, D. J. Hardy, N. L. Shipkowitz, C. W. Hanson, N. C. Ramer, P. B. Fernandes, J. J. Clement, Antimicrob. Agents Chemother. 1991, 35, 1108, doi: 10.1128/AAC.35.6.1108; b) D. J. Biedenbach, J. E. Ross, S. D. Putnam, R. N. Jones, Antimicrob. Agents Chemother. 2010, 54, 2273, doi: 10.1128/AAC.00090-10.

[10] F. Babakhani, J. Seddon, P. Sears, Antimicrob. Agents Chemother. 2014, 58, 2934, doi: 10.1128/AAC.02572-13.

[11] a) European Medicines Agency, Assessment Report Dificlir, 2011; b) Y. K. Shue, P. S. Sears, S. Shangle, R. B. Walsh, C. Lee, S. L. Gorbach, F. Okumu, R. A. Preston, Antimicrob. Agents Chemother. 2008, 52, 1391, doi: 10.1128/ AAC.01045-07.

[12] World Health Organization, Global Tuberculosis Report, 2019

[13] S. Sergio, G. Pirali, R. White, F. Parenti, J. Antibiot. 1975, 28, 543, doi: 10.7164/antibiotics.28.543

[14] a) M. Talpaert, F. Campagnari, L. Clerici, Biochem. Biophys. Res. Commun. 1975, 63, 328, doi: 10.1016/S0006-291X(75)80047-7; b) A. L. Sonenshein, H. B. Alexander, D. M. Rothstein, S. H. Fisher, J. Bacteriol. 1977, 132, 73; c) A. L. Sonenshein, H. B. Alexander, J. Mol. Biol. 1979, 127, 55, doi: 10.1016/0022-2836(79)90459-5; d) M. Gualtieri, P. Villain-Guillot, J. Latouche, J.-P. Leonetti, L. Bastide, Antimicrob. Agents Chemother. 2006, 50, 401, doi: 10.1128/AAC.50.1.401; e) A. Tupin, M. Gualtieri, J. P. Leonetti, K. Brodolin, EMBO J. 2010, 29, 2527, doi: 10.1038/emboj.2010.135; f) R. H. Ebright, WO Patent Appl. No. WO 2005/001034, 2005; g) A. Srivastava, M. Talaue, S. Liu, D. Degen, R. Y. Ebright, E. Sineva, A. Chakraborty, S. Y. Druzhinin, S. Chatterjee, J. Mukhopadhyay, Y. W. Ebright, A. Zozula, J. Shen, S. Sengupta, R. R. Niedfeldt, C. Xin, T. Kaneko, H. Irschik, R. Jansen, S. Donadio, N. Connell, R. H. Ebright, Curr. Opin. Microbiol. 2011, 14, 532, doi: 10.1016/j.mib.2011.07.030; h) R. H. Ebright, WO Patent Appl. No. WO 2007/094799 A1, 2006; i) Z. Morichaud, L. Chaloin, K. Brodolin, J. Mol. Biol. 2016, 428, 463, doi: 10.1016/j.jmb.2015.12.017.

[15] a) H. Boyaci, J. Chen, M. Lilic, M. Palka, R. A. Mooney, R. Landick, S. A. Darst, E. A. Campbell, Elife 2018, 7, 1, doi: 10.7554/eLife.34823; b) W. Lin, K. Das, D. Degen, C. Zhang, R. H. Ebright, W. Lin, K. Das, D. Degen, A. Mazumder, D. Duchi, D. Wang, Y. W. Ebright, Mol. Cell 2018, 70, 60, doi: 10.1016/j.molcel.2018.02.026.

[16] a) I. Artsimovitch, J. Seddon, P. Sears, Clin. Infect. Dis. 2012, 55, 127, doi: $10.1093 /$ cid/cis358 ; b) K. S. Murakami, S. Masuda, S. A. Darst, Science 2002, 296, 1280, doi: 10.1126/science.1069594 ; c) H. Mosaei, J. Harbottle, Biochem. Soc. Trans. 2019, 47, 339, doi: 10.1042/BST20180499.

[17] Y. Xiao, S. Li, S. Niu, L. Ma, G. Zhang, H. Zhang, G. Zhang, J. Ju, C. Zhang, J. Am. Chem. Soc. 2011, 133, 1092, doi: 10.1021/ja109445q.

[18] a) S. Niu, T. Hu, S. Li, Y. Xiao, L. Ma, G. Zhang, H. Zhang, X. Yang, J. Ju, C. Zhang, ChemBioChem 2011, 12, 1740, doi: 10.1002/cbic.201100129; b) H. Zhang, X. Tian, X. Pu, Q. Zhang, W. Zhang, C. Zhang, J. Nat. Prod. 2018, 81, 1219, doi: 10.1021/acs.jnatprod.7b00990; c) Z. Yu, H. Zhang, C. Yuan, Q. Zhang, I. Khan, Y. Zhu, C. Zhang, Org. Lett. 2019, 21, 7679, doi: 10.1021/acs.orglett.9b03100.

[19] A. Arnone, G. Nasini, B. Cavalleri, J. Chem. Soc. Perkin Trans 1 1987, 1353.

[20] a) J. E. McAlpine, J. E. Hochlowski, US Patent Appl. No. US 5,583,115, 1996; b) M.-C. Wu, C.-C. Huang, Y.-C. Lu, W.-J. Fan, US Patent Appl. No. US 2009/0110718 A1, 2008.

[21] F. Babakhani, A. Gomez, N. Robert, P. Sears, J. Med. Microbiol. 2011, 60, 1213, doi: 10.1099/jmm.0.029470-0.

\section{License and Terms}

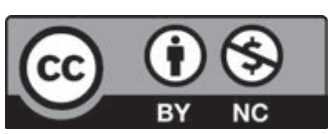

This is an Open Access article under the terms of the Creative Commons Attribution License CC BY_NC 4.0. The material may not be used for commercial purposes.

The license is subject to the CHIMIA terms and conditions: (http:// chimia.ch/component/sppagebuilder/?view=page \&id=12).

The definitive version of this article is the electronic one that can be found at doi:10.2533/chimia.2020.270 